\title{
Asymptotic Series of General Symbol of Pseudo-Differential Operator Involving Fractional Fourier Transform
}

\author{
S. K. Upadhyay, ${ }^{1}$ Anuj Kumar, ${ }^{2}$ and Jitendra Kumar Dubey ${ }^{2}$ \\ ${ }^{1}$ DST-CIMS, Department of Applied Mathematics, Indian Institute of Technology, Banaras Hindu University, Varanasi 221005, India \\ ${ }^{2}$ DST-CIMS, Banaras Hindu University, Varanasi 221005, India
}

Correspondence should be addressed to Anuj Kumar; anujk743@gmail.com

Received 16 July 2013; Accepted 13 August 2013

Academic Editors: T. Ozawa, W. Yu, and C. Zhu

Copyright (c) 2013 S. K. Upadhyay et al. This is an open access article distributed under the Creative Commons Attribution License, which permits unrestricted use, distribution, and reproduction in any medium, provided the original work is properly cited.

An asymptotic series of general symbol of pseudo-differential operator is obtained by using the theory of fractional Fourier transform.

\section{Introduction}

Namias [1] introduced fractional Fourier transform which is a generalization of Fourier transform. Fractional Fourier transform is the most important tool, which is frequently used in signal processing and other branches of mathematical sciences and engineering. The fractional Fourier transform can be considered as a rotation by an angle $\alpha$ in timefrequency plane and is also called rotational Fourier transform or angular Fourier transform. The fractional Fourier transform $[2,3]$, with angle $\alpha$ of a function $u(x)$, is defined by

$$
\widehat{u}_{\alpha}(x)=\left(F_{\alpha} u\right)(\xi)=\int_{\mathbb{R}} K_{\alpha}(x, \xi) u(x) d x,
$$

where

$$
\begin{gathered}
K_{\alpha}(x, \xi)=\left\{\begin{array}{ll}
C_{\alpha} e^{i\left(x^{2}+\xi^{2}\right) \cot \alpha / 2-i x \xi \operatorname{cosec} \alpha} & \text { if } \alpha \neq n \pi \\
\frac{1}{\sqrt{2 \pi}} e^{-i x \xi} & \text { if } \alpha=\frac{\pi}{2},
\end{array} \quad \forall n \in \mathbb{I},\right. \\
C_{\alpha}=(2 \pi i \sin \alpha)^{-1 / 2} e^{i \alpha / 2}=\sqrt{\frac{1-i \cot \alpha}{2 \pi}} .
\end{gathered}
$$

The corresponding inversion formula is given by

$$
u(x)=\frac{1}{2 \pi} \int_{\mathbb{R}} \overline{K_{\alpha}(x, \xi)} \widehat{u}_{\alpha}(\xi) d \xi,
$$

where the kernel

$$
\begin{aligned}
& \overline{K_{\alpha}(x, \xi)} \\
& =\left\{\begin{array}{ll}
C_{\alpha}^{\prime} e^{-i\left(x^{2}+\xi^{2}\right) \cot \alpha / 2+i x \xi \operatorname{cosec} \alpha} & \text { if } \alpha \neq n \pi \\
\frac{1}{\sqrt{2 \pi}} e^{i x \xi} & \text { if } \alpha=\frac{\pi}{2},
\end{array} \quad \forall n \in \mathbb{V},\right. \\
& C_{\alpha}^{\prime}=\frac{(2 \pi i \sin \alpha)^{1 / 2}}{\sin \alpha} e^{-i \alpha / 2}=\sqrt{2 \pi(1+i \cot \alpha)} .
\end{aligned}
$$

Zayed [3] and Bhosale and Chaudhary [4] studied fractional Fourier transform of distributions with compact support. Pathak and others [5] defined the pseudo-differential operator involving fractional Fourier transform on Schwartz space $S(\mathbb{R})$ and studied many properties.

Our main aim in this paper is to generalize the results of Zaidman [6] and to find an asymptotic series of general symbol of pseudo-differential operator involving fractional Fourier transform.

Now we are giving some definitions and properties which are useful for our further investigations.

Linearity of fractional Fourier transform is given as

$$
F_{\alpha}\left[c_{1} u_{1}(x)+c_{2} u_{2}(x)\right]=c_{1} F_{\alpha}\left(u_{1}(x)\right)+c_{2} F_{\alpha}\left(u_{2}(x)\right) \text {, }
$$

where $c_{1}$ and $c_{2}$ are constants and $u_{1}(x)$ and $u_{2}(x)$ are two input functions. 
Let $L^{p}(\mathbb{R})$ denote the class of measurable functions $f$ defined on $\mathbb{R}$ such that

$$
\|f\|_{L^{p}(\mathbb{R})}=\left[\int_{\mathbb{R}}|f(x)|^{p} d x\right]^{1 / p}<\infty,
$$

where $1 \leq p<\infty$.

From [5], generalized Sobolev space $\mathscr{G}_{\alpha}^{s, p}(\mathbb{R})$ involving fractional Fourier transform is defined by

$$
\|u\|_{s, p}=\left[\int_{\mathbb{R}}\left(1+|\xi|^{2}\right)^{s p / 2}\left|\widehat{u}_{\alpha}(\xi)\right|^{p} d \xi\right]^{1 / p}
$$

$s \in \mathbb{R}$ and $u \in S^{\prime}(\mathbb{R})$.

The convolution of two functions $f \in L^{1}(\mathbb{R})$ and $g \in$ $L^{1}(\mathbb{R})$ is defined $[5,7]$ as

$$
(f * g)(x)=\int_{\mathbb{R}} f(y) g(x-y) d y,
$$

provided that the integral exists.

Let $\mathscr{G}$ be a class of all measurable complex-valued functions $a(x, \xi)$ which are defined on $\mathbb{R} \times \mathbb{R}-\{0\}$. Then, we assume the following properties.

(i) $\lim _{x \rightarrow \infty} a(x, \xi)=a(\infty, \xi)$ exists for all $\xi \in \mathbb{R}-\{0\}$ and is bounded to mesaurable function.

(ii) We define $a^{\prime}(x, \xi)=a(x, \xi)-a(\infty, \xi)$, then

$$
\begin{array}{r}
a^{\prime}(x, \xi)=\frac{C_{\alpha}^{\prime}}{2 \pi} \int_{\mathbb{R}} e^{-i\left(x^{2}+\xi^{2}\right) \cot \alpha / 2+i x \xi \operatorname{cosec} \alpha}\left(F_{\alpha} a^{\prime}\right)(\eta, \xi) d \eta, \\
\forall(x, \xi) \in \mathbb{R} \times \mathbb{R}-\{0\},
\end{array}
$$

where $\left(F_{\alpha} a^{\prime}\right)(\eta, \xi)$ is complex-valued function defined on $\mathbb{R} \times$ $\mathbb{R}-\{0\}$, which is measurable in $\eta$ and $\xi$ for all $(\eta, \xi) \in \mathbb{R} \times \mathbb{R}-$ $\{0\}$ and satisfies the estimate:

$$
\left|\left(F_{\alpha} a^{\prime}\right)(\eta, \xi)\right| \leq K(\eta), \quad \forall \eta \in \mathbb{R},
$$

where $\left(1+|\eta|^{2}\right)^{l} K(\eta) \in L^{1}(\mathbb{R}) \forall l=0,1,2,3, \ldots$

Let $\left(r_{j}\right)_{0}^{\infty}$ be a strictly decreasing sequence; that is, $r_{0}>$ $r_{1}>r_{2}>\cdots>r_{j} \rightarrow-\infty$ as $j \rightarrow \infty$ and $\psi \in C^{\infty}(\mathbb{R})$ such that $0 \leq \psi(\xi) \leq \infty$ for all $\xi \in \mathbb{R}-\{0\}$,

$$
\psi(\xi)= \begin{cases}0 & \text { if } 0<\xi \leq \frac{1}{2} \\ 1 & \text { if } \xi \geq 1 .\end{cases}
$$

Let $\left\{a_{j}(x, \xi)\right\}_{0}^{\infty}$ be an infinite sequence of function defined on $\mathbb{R} \times \mathbb{R}-\{0\}$.

Then, we define a function

$$
a(x, \xi)=\sum_{j=0}^{\infty} \psi\left(\frac{\xi}{t_{j}}\right)|\xi|^{r_{j}} a_{j}(x, \xi),
$$

where $\left(t_{j}\right)_{0}^{\infty}$ is a sequence of positive real numbers such that $t_{j} \rightarrow \infty$ as $j \rightarrow \infty$.
From (12), it is clear that $a(x, \xi)=0$, for $|\xi| \leq 1 / 2, x \in \mathbb{R}$, and $t \in[1, \infty)$.

The global estimate of the above defined function $a(x, \xi)$ and of remainders of order $N$ is given as

$$
\begin{aligned}
b_{N}(x, \xi) & =a(x, \xi)-\sum_{j=0}^{N-1} \psi\left(\frac{\xi}{t_{j}}\right)|\xi|^{r_{j}} a_{j}(x, \xi) \\
& =\sum_{j=N}^{\infty} \psi\left(\frac{\xi}{t_{j}}\right)|\xi|^{r_{j}} a_{j}(x, \xi), \quad \forall \xi \in \mathbb{R}-\{0\} .
\end{aligned}
$$

Theorem 1. Let $\left\{t_{j}\right\}_{0}^{\infty}$ be a sequence of positive real numbers such that the following inequalities:

$$
\begin{aligned}
& |a(x, \xi)| \leq C|\xi|^{r_{0}}, \\
& \left|b_{N}(x, \xi)\right| \leq C|\xi|^{r_{N}}, \quad \forall(x, \xi) \in \mathbb{R} \times \mathbb{R}-\{0\},
\end{aligned}
$$

are satisfied for $N=1,2,3 \ldots$. In particular the estimates are as follows:

$$
\begin{array}{r}
|a(\infty, \xi)| \leq C|\xi|^{r_{0}}, \quad\left|b_{N}(\infty, \xi)\right| \leq C|\xi|^{r_{N}}, \\
\forall \xi \in \mathbb{R}-\{0\} .
\end{array}
$$

Proof. The proof of the above theorem is obvious from [6, pages 233-234].

Theorem 2. Let $\left\{t_{j}\right\}_{0}^{\infty}$ be a sequence of positive real numbers such that the following estimates:

$$
\begin{aligned}
& \left|\left(F_{\alpha} a^{\prime}\right)(x, \xi)\right| \leq K(\lambda)|\xi|^{r_{0}}, \\
& \left|\left(F_{\alpha} b_{N}^{\prime}\right)(x, \xi)\right| \leq K(\lambda)|\xi|^{r_{N}}, \quad \forall(\lambda, \xi) \in \mathbb{R} \times \mathbb{R}-\{0\},
\end{aligned}
$$

are satisfied for $N=1,2,3 \ldots$ In particular the estimates are as follows:

$$
\begin{array}{r}
\left|\left(F_{\alpha} a^{\prime}\right)(\infty, \xi)\right| \leq C|\xi|^{r_{0},} \quad\left|\left(F_{\alpha} b_{N}^{\prime}\right)(\infty, \xi)\right| \leq C|\xi|^{r_{N}}, \\
\forall \xi \in \mathbb{R}-\{0\},
\end{array}
$$

where $\left(1+|\lambda|^{2}\right)^{p} K(\lambda) \in L^{1}(\mathbb{R}),\left(1+|\lambda|^{2}\right)^{p} K_{N}(\lambda) \in L^{1}(\mathbb{R})$, $\forall p=0,1,2,3 \ldots$, and $N=1,2,3, \ldots$.

Proof. The proof of above theorem is also obvious by using the same arguments from [6, pages 133-135].

\section{Asymptotic Expansion of Pseudo-Differential Operator Associated with General Symbol}

Definition 3. Let $a(x, \xi)$ be a general symbol belonging to $\mathscr{G}$. Then pseudo-differential operator $a(x, D)=A_{a, \alpha}$ associated with symbol $a(x, \xi)$ is defined by

$$
(a(x, \xi) u)(x)=\frac{1}{2 \pi} \int_{\mathbb{R}} \overline{K_{\alpha}(x, \xi)} a(x, \xi) \widehat{u}_{\alpha}(\xi) d \xi,
$$

where $\widehat{u}_{\alpha}(\xi)$ is defined in $(3) \forall(x, \xi) \in \mathbb{R} \times \mathbb{R}-\{0\}$. 
Definition 4. An infinitely differentiable complex-valued function $u$ is member of $S(\mathbb{R})$ if and only if for every choice of $\mu$ and $\nu$ of non-negative integers, it satisfies

$$
\gamma_{\mu, \nu}(u)=\sup _{x \in \mathbb{R}}\left|x^{\mu} D^{\nu} u(x)\right|<\infty
$$

Lemma 5. A function $u \in C^{\infty}(\mathbb{R})$ satisfies (19) if and only if

$$
\tau_{\mu, \nu}(u)=\sup _{x \in \mathbb{R}}\left|\left(1+|x|^{2}\right)^{m / 2} D^{\beta} u(x)\right|<\infty, \quad \forall m, \beta \in N_{0} .
$$

Lemma 6 (Peetre). For any real number $t$ and for all $\xi, \eta \in \mathbb{R}$, the estimate

$$
\left(\frac{\left(1+|\xi|^{2}\right)}{\left(1+|\eta|^{2}\right)}\right)^{t} \leq 2^{|t|}(1+|\xi-\eta|)^{|t|}
$$

is satisfied.

Theorem 7. Let $a^{\prime}(x, \xi) \in \mathscr{G}$; then one has the following relation:

$$
\begin{aligned}
& F_{\alpha}\left[e^{i x^{2} \cot \alpha / 2} a^{\prime}(x, D) u(x)\right](\xi) \\
& =\frac{C_{\alpha}^{\prime}}{2 \pi} \int_{\mathbb{R}} e^{-i\left(\eta^{2}-\xi \eta\right) \cot \alpha} \widehat{a}_{\alpha}^{\prime}(\xi-\eta, \xi) \widehat{u}_{\alpha}(\eta) d \eta
\end{aligned}
$$

where $u \in S(\mathbb{R}), x \in \mathbb{R}$.

Proof. By the definition of fractional Fourier transform (1), we have

$$
\begin{aligned}
& F_{\alpha}\left[e^{i x^{2} \cot \alpha / 2} a^{\prime}(x, D) u(x)\right](\xi) \\
& =C_{\alpha} \int_{\mathbb{R}} e^{i\left(x^{2}+\xi^{2}\right) \cot \alpha / 2-i x \xi \operatorname{cosec} \alpha} \\
& \quad \times a^{\prime}(x, \xi) e^{i x^{2} \cot \alpha / 2} u(x) d x \\
& =C_{\alpha} \int_{\mathbb{R}} e^{i\left(x^{2}+\xi^{2}\right) \cot \alpha / 2-i x \xi \operatorname{cosec} \alpha} a^{\prime}(x, \xi) e^{i x^{2} \cot \alpha / 2} \\
& \quad \times\left\{\frac{C_{\alpha}^{\prime}}{2 \pi} \int_{\mathbb{R}} e^{-i\left(x^{2}+\eta^{2}\right) \cot \alpha / 2+i x \eta \operatorname{cosec} \alpha} \widehat{u}_{\alpha}(\eta) d \eta\right\} d x \\
& =\frac{C_{\alpha} C_{\alpha}^{\prime}}{2 \pi} \iint_{\mathbb{R}} e^{i\left(x^{2}+\xi^{2}-x^{2}-\eta^{2}+x^{2}\right) \cot \alpha / 2-i x(\xi-\eta) \operatorname{cosec} \alpha} \\
& \times a^{\prime}(x, \xi) \widehat{u}_{\alpha}(\eta) d \eta d x \\
& =\frac{C_{\alpha} C_{\alpha}^{\prime}}{2 \pi} \iint_{\mathbb{R}} e^{i\left(x^{2}+\xi^{2}-\eta^{2}\right) \cot \alpha / 2-i x(\xi-\eta) \operatorname{cosec} \alpha} \\
& \times a^{\prime}(x, \xi) \widehat{u}_{\alpha}(\eta) d \eta d x
\end{aligned}
$$

$$
\begin{gathered}
=\frac{C_{\alpha} C_{\alpha}^{\prime}}{2 \pi} \iint_{\mathbb{R}} e^{i\left(x^{2}+\xi^{2}+\eta^{2}-2 \xi \eta\right) \cot \alpha / 2-i x(\xi-\eta) \operatorname{cosec} \alpha} a^{\prime}(x, \xi) \\
\quad \times e^{i\left(\xi \eta-\eta^{2}\right) \cot \alpha} \widehat{u}_{\alpha}(\eta) d \eta d x \\
=\frac{C_{\alpha} C_{\alpha}^{\prime}}{2 \pi} \iint_{\mathbb{R}} e^{i\left(x^{2}+\xi^{2}+\eta^{2}-2 \xi \eta\right) \cot \alpha / 2-i x(\xi-\eta) \operatorname{cosec} \alpha} a^{\prime}(x, \xi) \\
\quad \times e^{i\left(\xi \eta-\eta^{2}\right) \cot \alpha} \widehat{u}_{\alpha}(\eta) d \eta d x \\
=\frac{C_{\alpha}^{\prime}}{2 \pi} \int_{\mathbb{R}} e^{-i\left(\eta^{2}-\xi \eta\right) \cot \alpha} \\
\quad \times\left\{C_{\alpha} \int_{\mathbb{R}} e^{i\left[x^{2}+(\xi-\eta)^{2}\right] \cot \alpha / 2-i x(\xi-\eta) \operatorname{cosec} \alpha}\right. \\
\left.\quad \times a^{\prime}(x, \xi) d x\right\} \widehat{u}_{\alpha}(\eta) d \eta \\
=\frac{C_{\alpha}^{\prime}}{2 \pi} \int_{\mathbb{R}} e^{-i\left(\eta^{2}-\xi \eta\right) \cot \alpha} \widehat{a}_{\alpha}^{\prime}(\xi-\eta, \xi) \widehat{u}_{\alpha}(\eta) d \eta .
\end{gathered}
$$

Theorem 8. If $a(x, \xi) \in \mathscr{G}$ is a symbol and $a(x, D)=$ $a(\infty, D)+a^{\prime}(x, D)$ is the associated operator, then, one has the following relation:

$$
\begin{aligned}
F_{\alpha}\left[e^{i x^{2} \cot \alpha / 2} a(x, D) u(x)\right](\xi) \\
=a(\infty, \xi) F_{\alpha}\left[e^{i x^{2} \cot \alpha / 2} u(x)\right](\xi) \\
\quad+\frac{C_{\alpha}^{\prime}}{2 \pi} \int_{\mathbb{R}} e^{-i\left(\eta^{2}-\xi \eta\right) \cot \alpha} \widehat{a}_{\alpha}^{\prime}(\xi-\eta, \xi) \widehat{u}_{\alpha}(\eta) d \eta,
\end{aligned}
$$

where $u \in S(\mathbb{R}), x \in \mathbb{R}$.

Proof. We have

$$
\begin{aligned}
F_{\alpha} & {\left[e^{i x^{2} \cot \alpha / 2} a(x, D) u(x)\right](\xi) } \\
& =F_{\alpha}\left[e^{i x^{2} \cot \alpha / 2}\left\{a(\infty, D)+a^{\prime}(x, D)\right\} u(x)\right](\xi) .
\end{aligned}
$$

By linearity of fractional Fourier transform (5) we get

$$
\begin{aligned}
& F_{\alpha}\left[e^{i x^{2} \cot \alpha / 2} a(x, D) u(x)\right](\xi) \\
&= F_{\alpha}\left[e^{i x^{2} \cot \alpha / 2} a(\infty, D) u(x)\right](\xi) \\
&+F_{\alpha}\left[e^{i x^{2} \cot \alpha / 2} a^{\prime}(x, D) u(x)\right](\xi) .
\end{aligned}
$$

Now using (1) and (22), we get the required result. 
Theorem 9. Let $a(x, \xi) \in \mathscr{G}$ be a symbol and $a(x, D)$ the associated operator; then one has the following relation:

$$
\|(a(x, D) u)\|_{s, p} \leq C_{s, p}\|u\|_{s+r_{0}, p},
$$

where $u \in S(\mathbb{R}), s \in \mathbb{R}$.

Proof. Firstly from (7) we have

$$
\begin{aligned}
& \left\|e^{i x^{2} \cot \alpha / 2}(a(\infty, D) u)\right\|_{s, p} \\
& =\left[\int_{\mathbb{R}}\left(1+|\xi|^{2}\right)^{s p / 2}\left|F_{\alpha}\left[e^{i x^{2} \cot \alpha / 2} a(\infty, D) u(x)\right](\xi)\right|^{p} d \xi\right]^{1 / p} \\
& =\left[\int_{\mathbb{R}}\left(1+|\xi|^{2}\right)^{s p / 2}|a(\infty, \xi)|^{p}\left|F_{\alpha}\left[e^{i x^{2} \cot \alpha / 2} u(x)\right](\xi)\right|^{p} d \xi\right]^{1 / p} .
\end{aligned}
$$

Now the argument of (15) yields

$$
\begin{gathered}
\left\|e^{i x^{2} \cot \alpha / 2}(a(\infty, D) u)\right\|_{s, p} \\
\leq C\left[\int_{\mathbb{R}}\left(1+|\xi|^{2}\right)^{s p / 2}\left(1+|\xi|^{2}\right)^{r_{0} p / 2}\right. \\
\left.\times\left|F_{\alpha}\left[e^{i x^{2} \cot \alpha / 2} u(x)\right](\xi)\right|^{p} d \xi\right]^{1 / p} \\
=C\left[\int_{\mathbb{R}}\left(1+|\xi|^{2}\right)^{\left(s+r_{0}\right) p / 2}\right. \\
\left.\quad \times\left|F_{\alpha}\left[e^{i x^{2} \cot \alpha / 2} u(x)\right](\xi)\right|^{p} d \xi\right]^{1 / p} \\
=C\left\|e^{i x^{2} \cot \alpha / 2} u\right\|_{s+r_{0}, p}
\end{gathered}
$$

Thus we have

$$
\begin{gathered}
\left\|e^{i x^{2} \cot \alpha / 2}(a(\infty, D) u)\right\|_{s, p} \leq C\left\|e^{i x^{2} \cot \alpha / 2} u\right\|_{s+r_{0}, p}, \\
\left\|e^{i x^{2} \cot \alpha / 2}(a(\infty, D) u)\right\|_{s, p} \leq C\|u\|_{s+r_{0}, p},
\end{gathered}
$$

for every $u \in S(\mathbb{R}), s \in \mathbb{R}$.

Now we consider the function $U_{s, \alpha}(\xi)=\left(1+|\xi|^{2}\right)^{s / 2} G_{\alpha}(\xi)$, where $U_{s, \alpha}(\xi)=0$ for $|\xi| \leq 1 / 2$ and for $|\xi| \geq 1 / 2$ and $G_{\alpha}(\xi)$ is defined as

$$
G_{s}(\xi)=\frac{C_{\alpha}^{\prime}}{2 \pi} \int_{\mathbb{R}} e^{-i\left(\eta^{2}-\xi \eta\right) \cot \alpha} \widehat{a}_{\alpha}^{\prime}(\xi-\eta, \xi) \widehat{u}_{\alpha}(\eta) d \eta .
$$

Therefore,

$$
\begin{aligned}
& U_{s, \alpha}(\xi) \\
& =\frac{C_{\alpha}^{\prime}}{2 \pi}\left(1+|\xi|^{2}\right)^{s / 2} \int_{\mathbb{R}} e^{-i\left(\eta^{2}-\xi \eta\right) \cot \alpha} \widehat{a}_{\alpha}^{\prime}(\xi-\eta, \xi) \widehat{u}_{\alpha}(\eta) d \eta \\
& =\frac{C_{\alpha}^{\prime}}{2 \pi} \int_{\mathbb{R}} \frac{\left(1+|\xi|^{2}\right)^{s / 2}}{\left(1+|\eta|^{2}\right)^{s / 2}} e^{-i\left(\eta^{2}-\xi \eta\right) \cot \alpha} \\
& \quad \times\left(1+|\eta|^{2}\right)^{s / 2} \widehat{a}_{\alpha}^{\prime}(\xi-\eta, \xi) \widehat{u}_{\alpha}(\eta) d \eta .
\end{aligned}
$$

Using (21) we get

$$
\begin{aligned}
\left|U_{s, \alpha}(\xi)\right| \leq \frac{2^{|s| / 2} C_{\alpha}^{\prime}}{2 \pi} \int_{\mathbb{R}} & \left(1+|\xi-\eta|^{2}\right)^{|s| / 2}\left(1+|\eta|^{2}\right)^{s / 2} \\
& \times\left|\widehat{a}_{\alpha}^{\prime}(\xi-\eta, \xi)\right|\left|\widehat{u}_{\alpha}(\eta)\right| d \eta .
\end{aligned}
$$

From Theorem 2 we get

$$
\begin{aligned}
& \left|U_{s, \alpha}(\xi)\right| \\
& \leq \frac{2^{|s| / 2} C_{\alpha}^{\prime}}{2 \pi} \int_{\mathbb{R}}\left(1+|\xi-\eta|^{2}\right)^{|s| / 2}\left(1+|\eta|^{2}\right)^{s / 2} \\
& \times K(\xi-\eta)\left(1+|\xi|^{2}\right)^{r_{0} / 2}\left|\widehat{u}_{\alpha}(\eta)\right| d \eta, \\
& =\frac{2^{|s| / 2} C_{\alpha}^{\prime}}{2 \pi} \int_{\mathbb{R}}\left(1+|\xi-\eta|^{2}\right)^{|s| / 2}\left(1+|\eta|^{2}\right)^{\left(s+r_{0}\right) / 2} \\
& \times K(\xi-\eta) \frac{\left(1+|\xi|^{2}\right)^{r_{0} / 2}}{\left(1+|\eta|^{2}\right)^{r_{0} / 2}}\left|\widehat{u}_{\alpha}(\eta)\right| d \eta, \\
& =\frac{2^{\left(|s|+\left|r_{0}\right|\right) / 2} C_{\alpha}^{\prime}}{2 \pi} \int_{\mathbb{R}}\left(1+|\xi-\eta|^{2}\right)^{|s| / 2}\left(1+|\xi-\eta|^{2}\right)^{\left|r_{0}\right| / 2} \\
& \times\left(1+|\eta|^{2}\right)^{\left(s+r_{0}\right) / 2} K(\xi-\eta)\left|\widehat{u}_{\alpha}(\eta)\right| d \eta, \\
& =\frac{2^{\left(|s|+\left|r_{0}\right|\right) / 2-1} C_{\alpha}^{\prime}}{\pi} \\
& \times \int_{\mathbb{R}}\left(1+|\xi-\eta|^{2}\right)^{\left(|s|+\left|r_{0}\right|\right) / 2}\left(1+|\eta|^{2}\right)^{\left(s+r_{0}\right) / 2} \\
& \times K(\xi-\eta)\left|\widehat{u}_{\alpha}(\eta)\right| d \eta, \\
& =\int_{\mathbb{R}} \Phi(\xi-\eta) h_{\alpha}(\eta) d \eta \\
& =\left(\Phi * h_{\alpha}\right)(\xi) \text {. }
\end{aligned}
$$

Here $\Phi(\xi-\eta) \in L^{1}(\mathbb{R})$ by (10) for all $s \in \mathbb{R}$ and since $\widehat{u}_{\alpha}(\eta) \epsilon$ $S(\mathbb{R})$, therefore $h_{\alpha}(\eta) \in L^{p}(\mathbb{R})$.

Thus, we have $\left(\Phi * h_{\alpha}\right)(\xi) \in L^{p}(\mathbb{R})$ and the inequality

$$
\left\|\Phi * h_{\alpha}\right\|_{L^{p}(\mathbb{R})} \leq\|\Phi\|_{L^{1}(\mathbb{R})}\left\|h_{\alpha}\right\|_{L^{p}(\mathbb{R})} .
$$

This implies that

$$
\left\|U_{s, \alpha}(\xi)\right\|_{p} \leq C_{\alpha}\|u\|_{s+r_{0}, p}, \quad \forall u \in S(\mathbb{R}), s \in \mathbb{R} .
$$

So that

$$
\begin{aligned}
& \left\|e^{i x^{2} \cot \alpha / 2}\left(a^{\prime}(x, D) u\right)\right\|_{s, p} \\
& =\left\|\left(1+|\xi|^{2}\right)^{s / 2} F_{\alpha}\left[e^{i x^{2} \cot \alpha / 2}\left(a^{\prime}(x, D) u\right)(x)\right]\right\|_{p} \\
& =\left\|U_{s, \alpha}(\xi)\right\|_{p} .
\end{aligned}
$$


Using (36) we get

$$
\left\|e^{i x^{2} \cot \alpha / 2}\left(a^{\prime}(x, D) u\right)\right\|_{s, p} \leq C_{\alpha}\|u\|_{s+r_{0}, p} .
$$

Therefore,

$$
\begin{gathered}
\left\|e^{i x^{2} \cot \alpha / 2}(a(x, D) u)\right\|_{s, p} \\
=\left\|e^{i x^{2} \cot \alpha / 2}\left\{a(\infty, D) u+a^{\prime}(x, D) u\right\}\right\|_{s, p} \\
\leq\left\|e^{i x^{2} \cot \alpha / 2}(a(\infty, D) u)\right\|_{s, p} \\
+\left\|e^{i x^{2} \cot \alpha / 2}\left(a^{\prime}(x, D) u\right)\right\|_{s, p} .
\end{gathered}
$$

Now using (30) and (38) we get

$$
\left\|e^{i x^{2} \cot \alpha / 2}(a(x, D) u)\right\|_{s, p} \leq\left(C+C_{\alpha}\right)\|u\|_{s+r_{0}, p} .
$$

This implies that

$$
\left\|e^{i x^{2} \cot \alpha / 2}(a(x, D) u)\right\|_{s, p} \leq C_{s, p, \alpha}\|u\|_{s+r_{0}, p} .
$$

This implies the required result (27).

Theorem 10. One has the following estimates:

$$
\left\|\psi_{r}\left(\frac{1}{t} D\right) u\right\|_{s, p} \leq C\|u\|_{s+r, p}, \quad \forall u \in S(\mathbb{R}), s, r \in \mathbb{R} .
$$

Proof. Let $\psi \in C^{\infty}(\mathbb{R})$, then by using (11) and (12) we find that $\psi_{r}(\xi / t)=\psi(\xi / t)|\xi|^{r}$ for $|\xi| \geq t / 2$. Therefore, we have

$$
\left|\psi_{r}\left(\frac{\xi}{t}\right)\right| \leq C\left(1+|\xi|^{2}\right)^{r / 2}, \quad \forall \xi \in \mathbb{R} .
$$

Now we define the operator $\psi_{r}((1 / t) D)$ given on $S(\mathbb{R})$ by

$$
\psi_{r}\left(\frac{1}{t} D\right) u=F_{\alpha}^{-1}\left(\psi_{r}\left(\frac{\xi}{t}\right) \widehat{u}_{\alpha}(\xi)\right) .
$$

Hence,

$$
\begin{aligned}
& \left\|\psi_{r}\left(\frac{1}{t} D\right) u\right\|_{s, p} \\
& \quad=\left(\int_{\mathbb{R}}\left(1+|\xi|^{2}\right)^{s p / 2}\left|\psi_{r}\left(\frac{\xi}{t}\right)\right|^{p}\left|\widehat{u}_{\alpha}(\xi)\right|^{p} d \xi\right)^{1 / p} \\
& \quad \leq C\left(\int_{\mathbb{R}}\left(1+|\xi|^{2}\right)^{s p / 2}\left|\widehat{u}_{\alpha}(\xi)\right|^{p}\left(1+|\xi|^{2}\right)^{r p / 2} d \xi\right)^{1 / p} \\
& \quad=C\left(\int_{\mathbb{R}}\left(1+|\xi|^{2}\right)^{(s+r) p / 2}\left|\widehat{u}_{\alpha}(\xi)\right|^{p} d \xi\right)^{1 / p} \cdot
\end{aligned}
$$

Thus,

$$
\left\|\psi_{r}\left(\frac{1}{t} D\right) u\right\|_{s, p} \leq C\|u\|_{s+r, p} .
$$

Since $\mathscr{G}_{\alpha}^{\infty}=\cap_{s \in \mathbb{R}} \mathscr{G}_{\alpha}^{s, p}(\mathbb{R}), \psi_{r}(((1 / t) D) u)$ is a linear operator $\mathscr{G}_{\alpha}^{\infty}$ into itself.
Definition 11. A linear operator $L$ with $r \in \mathbb{R}$, and $\forall s \in \mathbb{R}$, there exists a constant $C_{s}>0$ such that

$$
\|L u\| \leq C_{s}\|u\|_{s+r, p}, \quad \forall u \in \mathscr{G}_{\alpha}^{\infty}
$$

The infimum of all orders $r$ of $L$ is called true order of $L$.

Definition 12. Let $\psi_{r}(((1 / t) D) a(x, D))$ be a linear operator from $\forall u \in \mathscr{G}_{\alpha}^{\infty}$ into itself and satisfy the following inequality:

$$
\left\|\psi_{r}\left(\frac{1}{t} D\right) a(x, D)\right\|_{s, p} \leq C\|u\|_{s+r, p}, \quad \forall s \in \mathbb{R}, u \in \mathscr{G}_{\alpha}^{\infty}(\mathbb{R}) .
$$

Then $\psi_{r}((1 / t) D) a(x, D)$ is said to be a canonical operator of degree $r$, where $r \in \mathbb{R}$.

Definition 13. Let $\left(r_{j}\right)_{0}^{\infty}$ be a strictly decreasing sequence of real numbers and $\left\{\psi_{r_{j}}((1 / t) D) a_{j}(x, D)\right\}$ a sequence of canonical operators of degree $r_{j}$. Then, corresponding a sequence of positive real numbers $\left(t_{j}\right)_{0}^{\infty}$ and $\left\{a_{j}(x, \xi)\right\}_{0}^{\infty} \subset \mathscr{G}$, a linear operator $M: \mathscr{G}_{\alpha}^{\infty} \rightarrow \mathscr{G}_{\alpha}^{\infty}$ is asymptotically expanded into the series $\sum_{j=0}^{\infty} \psi_{r_{j}}((1 / t) D) a_{j}(x, D)$ if it satisfies the following inequality:

$$
\text { t.o }\left[M-\sum_{j=0}^{N} \psi_{r_{j}}\left(\frac{1}{t_{j}} D\right) a_{j}(x, D)\right]<r_{N} \text {. }
$$

Theorem 14. Let $\left\{a_{j}(x, \xi)\right\}_{0}^{\infty}$ be a sequence of symbols belonging to $\mathscr{G}$ and $\left(r_{j}\right)_{0}^{\infty}$ a strictly decreasing sequence of real numbers that tends to $-\infty$. Then, there exists a sequence of canonical operators of degree $r_{j}, K_{j, \alpha}$ and a linear operator $A_{a, \alpha}$ in $\mathscr{G}_{\alpha}^{\infty}$ such that

(i) t.o $\left(A_{a, \alpha}\right) \leq r_{0}$;

(ii) $A_{a, \alpha} \sim \sum_{j=o}^{\infty} K_{j, \alpha}$, that is, t.o $\left[A_{a, \alpha}-\sum_{j=o}^{N} K_{j, \alpha}\right]<r_{N}$.

Proof. Note that (i) is obviously true by using Theorem 9 and for (ii) by using the arguments of [6, pages 241-242]. We can define the canonical operator by the following way:

$$
\begin{aligned}
& \left(K_{j, \alpha} u\right)(x) \\
& =e^{-i x^{2} \cot \alpha / 2} F_{\alpha}^{-1} \\
& \quad \times\left[\psi _ { r _ { j } } ( \frac { \xi } { t _ { j } } ) \left\{a_{j}(\infty, \xi) F_{\alpha}\left(e^{i x^{2} \cot \alpha / 2} u(x)\right)(\xi)\right.\right. \\
& \quad+\frac{C_{\alpha}^{\prime}}{2 \pi} \int_{\mathbb{R}} e^{-i\left(\eta^{2}-\xi \eta\right) \cot \alpha} \\
& \left.\left.\quad \times \hat{a}_{j, \alpha}^{\prime}(\xi-\eta, \xi) \widehat{u}_{\alpha}(\eta) d \eta\right\}\right],
\end{aligned}
$$


where $a(x, \xi)=\sum_{j=o}^{\infty} \psi_{r_{j}}(\xi / t)\left[a_{j}(\infty, \xi)+a_{j}^{\prime}(x, \xi)\right]$ belongs to class of symbol $\mathscr{G}$. Also we have

$$
\begin{aligned}
\left(A_{a, \alpha} u\right)(x)= & e^{-i x^{2} \cot \alpha / 2} F_{\alpha}^{-1} \\
& \times\left[a(\infty, \xi) F_{\alpha}\left\{e^{i x^{2} \cot \alpha / 2} u(x)\right\}(\xi)\right. \\
& +\frac{C_{\alpha}^{\prime}}{2 \pi} \int_{\mathbb{R}} e^{-i\left(\eta^{2}-\xi \eta\right) \cot \alpha} \\
& \left.\times \hat{a}_{\alpha}^{\prime}(\xi-\eta, \xi) \widehat{u}_{\alpha}(\eta) d \eta\right] .
\end{aligned}
$$

Therefore,

$$
\begin{aligned}
F_{\alpha}\left[e^{i x^{2} \cot \alpha / 2}\left(A_{a, \alpha} u-\sum_{j=0}^{\infty} K_{j, \alpha} u\right)\right](\xi) \\
=\left[\left\{a(\infty, \xi)-\sum_{j=0}^{N} \psi_{r_{j}}\left(\frac{\xi}{t_{j}}\right) a_{j}(\infty, \xi)\right\}\right. \\
\times F_{\alpha}\left\{e^{i x^{2} \cot \alpha / 2} u(x)\right\}(\xi) \\
+\frac{C_{\alpha}^{\prime}}{2 \pi} \int_{\mathbb{R}} e^{-i\left(\eta^{2}-\xi \eta\right) \cot \alpha} \\
\times\left\{\widehat{a}_{\alpha}^{\prime}(\xi-\eta, \xi)-\sum_{j=0}^{N} \psi_{r_{j}}\left(\frac{\xi}{t_{j}}\right) \widehat{a}_{j, \alpha}^{\prime}(\xi-\eta, \xi)\right\} \\
\left.\quad \times \widehat{u}_{\alpha}(\eta) d \eta\right]
\end{aligned}
$$

Using the previous definitions (12) and (13), we have

$$
a(\infty, \xi)-\sum_{0}^{N} \psi_{r_{j}}\left(\frac{\xi}{t_{j}}\right) a_{j}(\infty, \xi)=b_{N+1}(\infty, \xi)
$$

From (15) and (16), we get $\left|b_{N+1}(\infty, \xi)\right| \leq C\left(1+|\xi|^{2}\right)^{r_{N+1} / 2}$, where $\xi \in \mathbb{R}-\{0\}$ and $\widehat{a}_{\alpha}^{\prime}(\xi-\eta, \xi)-\sum_{j=0}^{N} \psi_{r_{j}}\left(\xi / t_{j}\right) \widehat{a}_{\alpha, j}(\xi-$ $\eta, \xi)=\widehat{b}_{\alpha, N+1}^{\prime}(\xi-\eta, \xi)$.

Also $\left|\widehat{b}_{\alpha}^{\prime}(\xi-\eta, \xi)\right| \leq C K_{N+1}(\xi-\eta)\left(1+|\xi|^{2}\right)\left(r_{N+1} / 2\right)$. Now using these arguments in (52) we get

$$
\begin{aligned}
F_{\alpha}\left[e^{i x^{2} \cot \alpha / 2}\left(A_{a, \alpha}-\sum_{j=0}^{N} K_{j, \alpha} u\right)\right](\xi) \\
=b_{N+1}(\infty, \xi) F_{\alpha}\left\{e^{i x^{2} \cot \alpha / 2} u(x)\right\}(\xi) \\
\quad+\frac{C_{\alpha}^{\prime}}{2 \pi} \int_{\mathbb{R}} e^{-i\left(\eta^{2}-\xi \eta\right) \cot \alpha} \widehat{b}_{\alpha}^{\prime}(\xi-\eta, \xi) \widehat{u}_{\alpha}(\eta) d \eta
\end{aligned}
$$

Using Theorem 9, we get

$$
\left\|A_{a, \alpha} u-\sum_{j=0}^{N} K_{j} u\right\|_{s, p} \leq C\|u\|_{s+r_{N+1}, p}, \quad \forall u \in S(\mathbb{R}), s \in \mathbb{R}
$$

Hence, we get

$$
\text { t.o }\left[A_{a, \alpha}-\sum_{j=0}^{N} K_{j, \alpha}\right] \leq r_{N+1}<r_{N}
$$

\section{Acknowledgments}

The first author is thankful to DST-CIMS, Banaras Hindu University, Varanasi, India, for providing the research facilities, and the second author is also thankful to DST-CIMS, Banaras Hindu University, Varanasi, India, for awarding the Junior Research Fellowship from December 2012.

\section{References}

[1] V. Namias, "The fractional order Fourier transform and its application to quantum mechanics," Journal of the Institute of Mathematics and its Applications, vol. 25, no. 3, pp. 241-265, 1980.

[2] L. M. Almeida, "The fractional Fourier transform and timefrequency representations," IEEE Signal Processing Letters, vol. 42, no. 11, pp. 3084-3091, 1994.

[3] A. I. Zayed, "Fractional Fourier transform of generalized functions," Integral Transforms and Special Functions, vol. 7, no. 3-4, pp. 299-312, 1998.

[4] B. N. Bhosale and M. S. Chaudhary, "Fractional Fourier transform of distributions of compact support," Bulletin of the Calcutta Mathematical Society, vol. 94, no. 5, pp. 349-358, 2002.

[5] R. S. Pathak, A. Prasad, and M. Kumar, "Fractional Fourier transform of tempered distributions and generalized pseudodifferential operator," Journal of Pseudo-Differential Operators and Applications, vol. 3, no. 2, pp. 239-254, 2012.

[6] S. Zaidman, "On asymptotic series of symbols and of general pseudodifferential operators," Rendiconti del Seminario Matematico dell'Università di Padova, vol. 63, pp. 231-246, 1980.

[7] R. S. Pathak, A Course in Distribution Theory and Applications, Narosa Publishing House, New Delhi, India, 2009. 


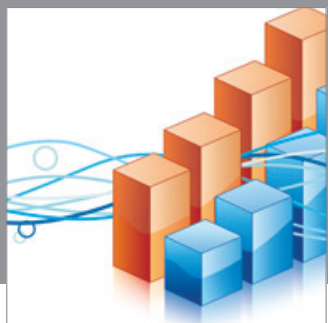

Advances in

Operations Research

mansans

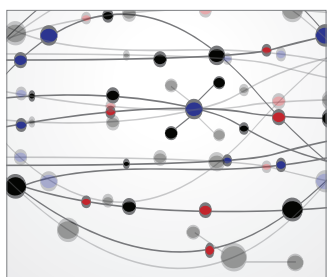

The Scientific World Journal
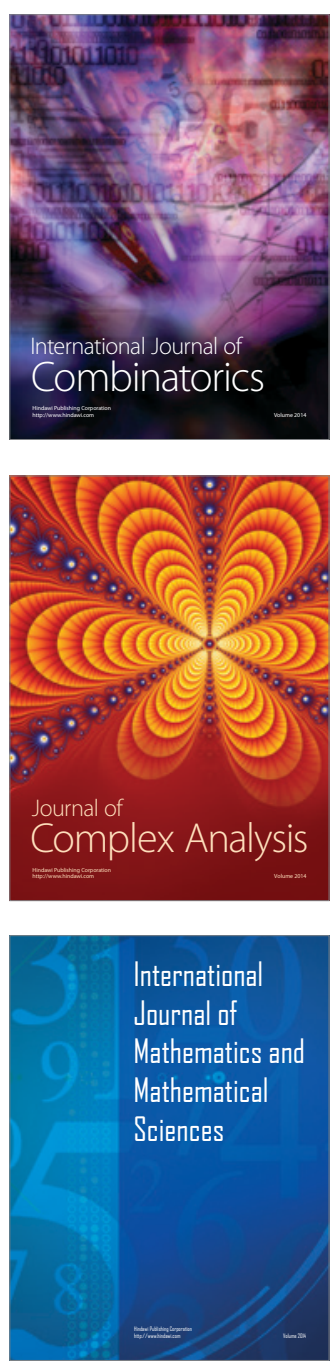
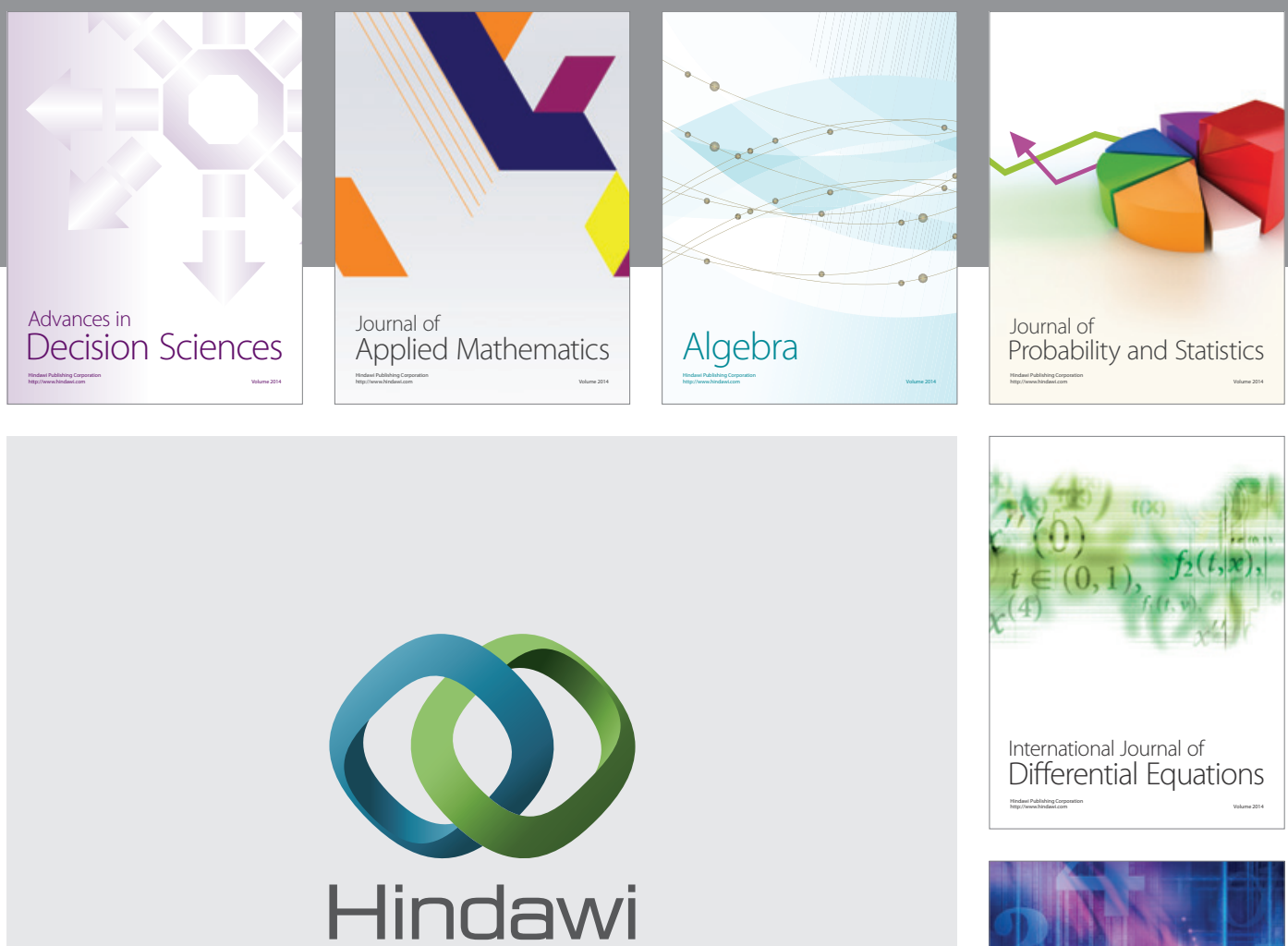

Submit your manuscripts at http://www.hindawi.com
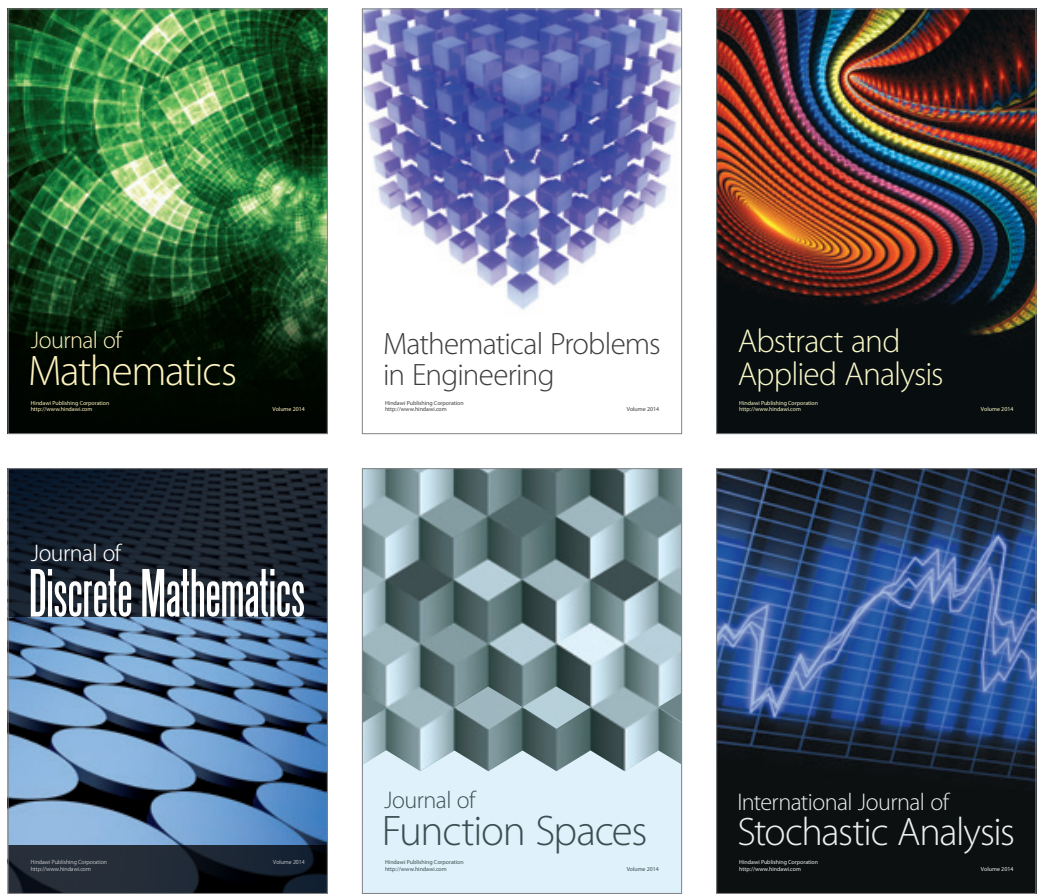

Journal of

Function Spaces

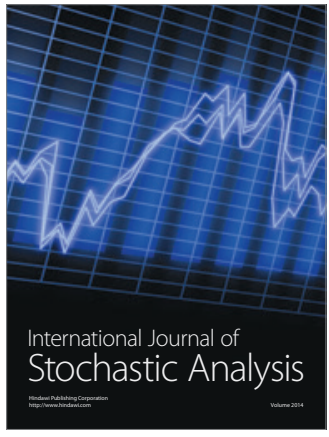

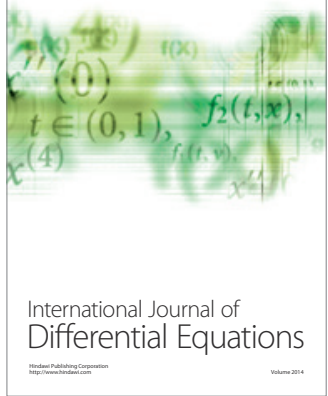
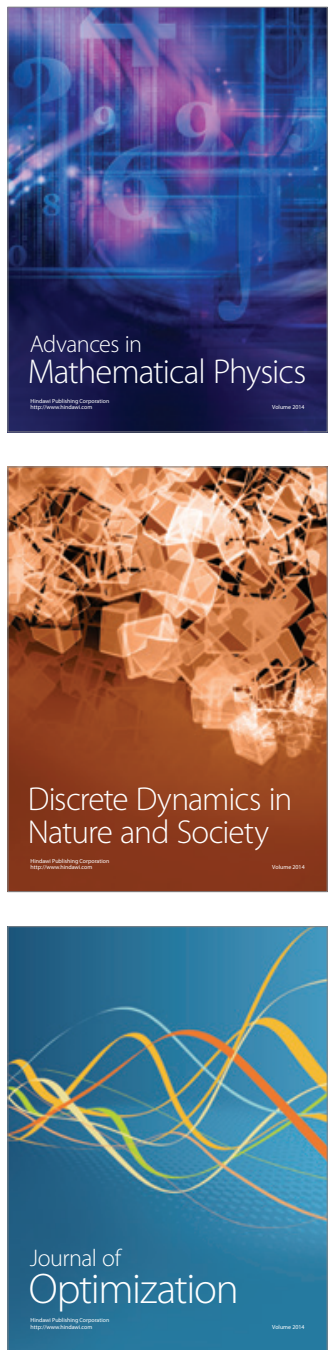